\title{
OCORRÊNCIA DE PARASTYGARCTUS STERRERI RENAUD-MORNANT, 1970 E HALECHINISCUS PERFECTUS SCHULZ, 1955 (TARDIGRADA, HETEROTARDIGRADA) NO ARQUIPÉLAGO DE SÃO PEDRO E SÃO PAULO, RN, BRASIL.
}

JULIANA DA ROCHA MOURA ${ }^{1,2,3}$, MÔNICA MARINHO VERÇOSA ${ }^{1,2}$, ÉRIKA CAVALCANTE LEITE DOS SANTOS ${ }^{1,2}$, LUÍZA GABRIELA
SANTANA E SILVA', FERNANDA M. DUARTE DO AMARAL', CLÉLIA MÁRCIA CAVALCANTI DA ROCHA'.
'Universidade Federal Rural de Pernambuco - Depto. de Biologia, Lab. de Meiofauna. R. Dom Manoel de Medeiros, s/n - Dois Irmãos, CEP: Universidade Federal Rural de Pernambico - Depto. de Be - Brasil. ${ }^{2}$ PIBIC/CNPq, ${ }^{3}$ julidrm@ @otmail.com.
$52171-900$ - Recife -PE

\section{RESUMO}

Amostragens da meiofauna feitas em substratos inconsolidados no Arquipélago de São Pedro e São Paulo resultaram na identificação e registro de duas espécies de Tardigrada ainda não observadas no Brasil: Parastygarctus sterreri RenaudMornant, 1970 e Halechiniscus perfectus Schulz, 1955. O presente estudo amplia para 11 o número de gêneros e 14 o número de espécies conhecidas no país.

PALAVRAS CHAVE: Tardigrada, illhas oceânicas, meiofauna

\section{ABSTRACT}

Occurrence of Parastygarctus sterreri Renaud-Mornant, 1970 and Halechiniscus perfectus Schulz, 1955 (Tardigrada, Heterotardigrada) at Saint Peter and St. Paul Archipelago, RN, Brazil.

Occurrence of Parastygarctus sterreri Renaud-Mornant, 1970 and Halechiniscus perfectus Schulz, 1955 (Tardigrada, Heterotardigrada) at Saint Peter and St. Paul Archipelago, RN, Brazil. Meiofaunistic samplings were carried out in sublittoral soft bottoms from Saint Peter and St. Paul Archipelago (RN, Brazil), resulting in the identification and recording of two species still non-observed in Brazil: Parastygarctus sterreri Renaud-Mornant, 1970 and Halechiniscus perfectus Schulz, 1955. The present study broadens to 11 the number of genera and to 14 the number of species in Brazil.

KEY WORDS: Tardigrada, oceanic islands, meiofauna

Amostragens da meiofauna realizadas no infralitoral do Arquipélago de São Pedro e São Paulo $\left(00^{\circ} 56^{\prime} \mathrm{N} ; 29^{\circ} 22^{\prime} \mathrm{W}\right)$ em março e agosto de 2007 propiciaram a identificação e registro de duas espécies de tardígrados marinhos até então não observadas no Brasil: Parastygarctus sterreri RenaudMornant, 1970 e Halechiniscus perfectus (Schulz, 1955).

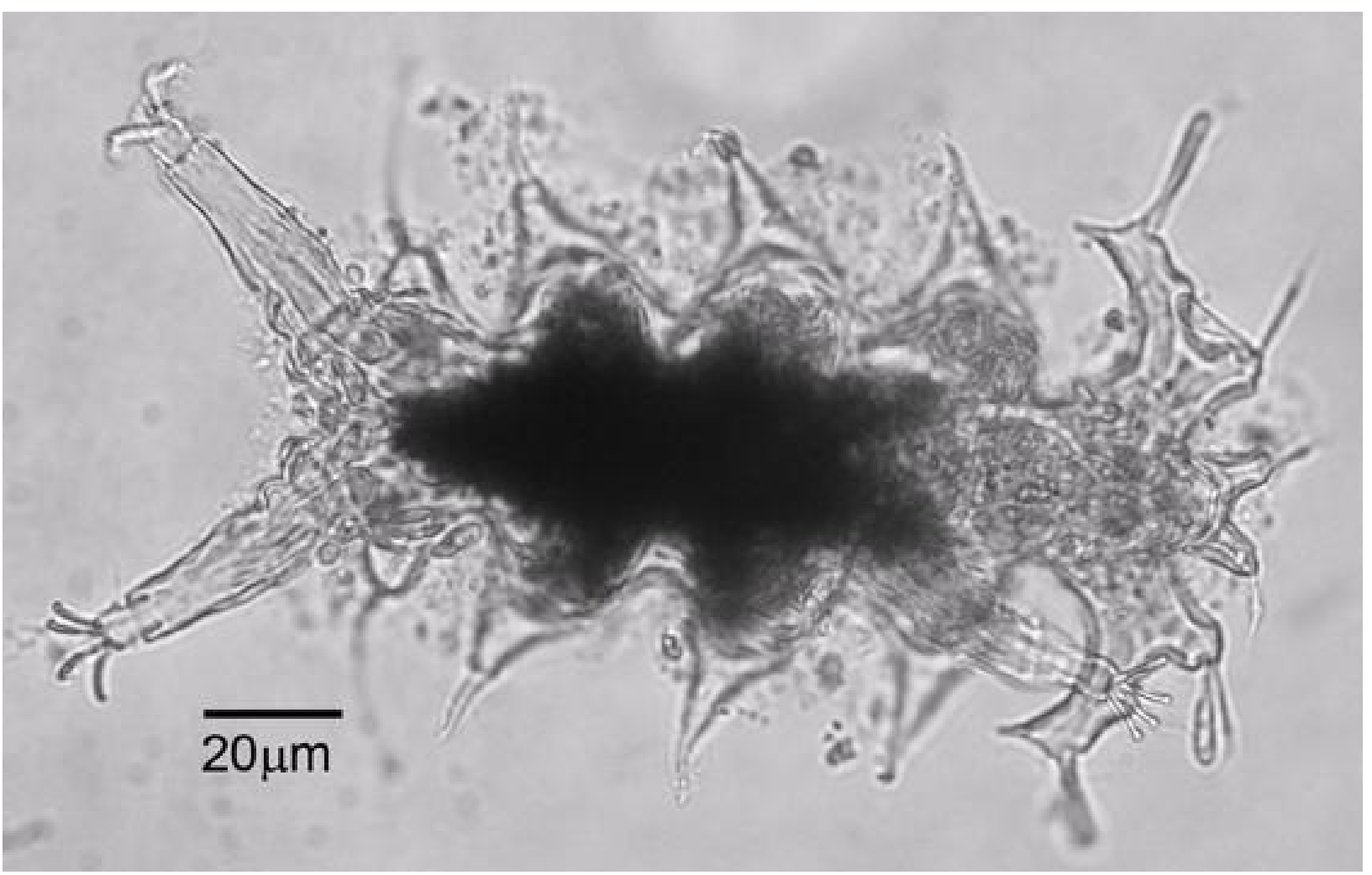

Figura 1 - Exemplar de Parastygarctus sterreri coletado no Arquipélago de São Pedro e São Paulo, RN, Brasil. 


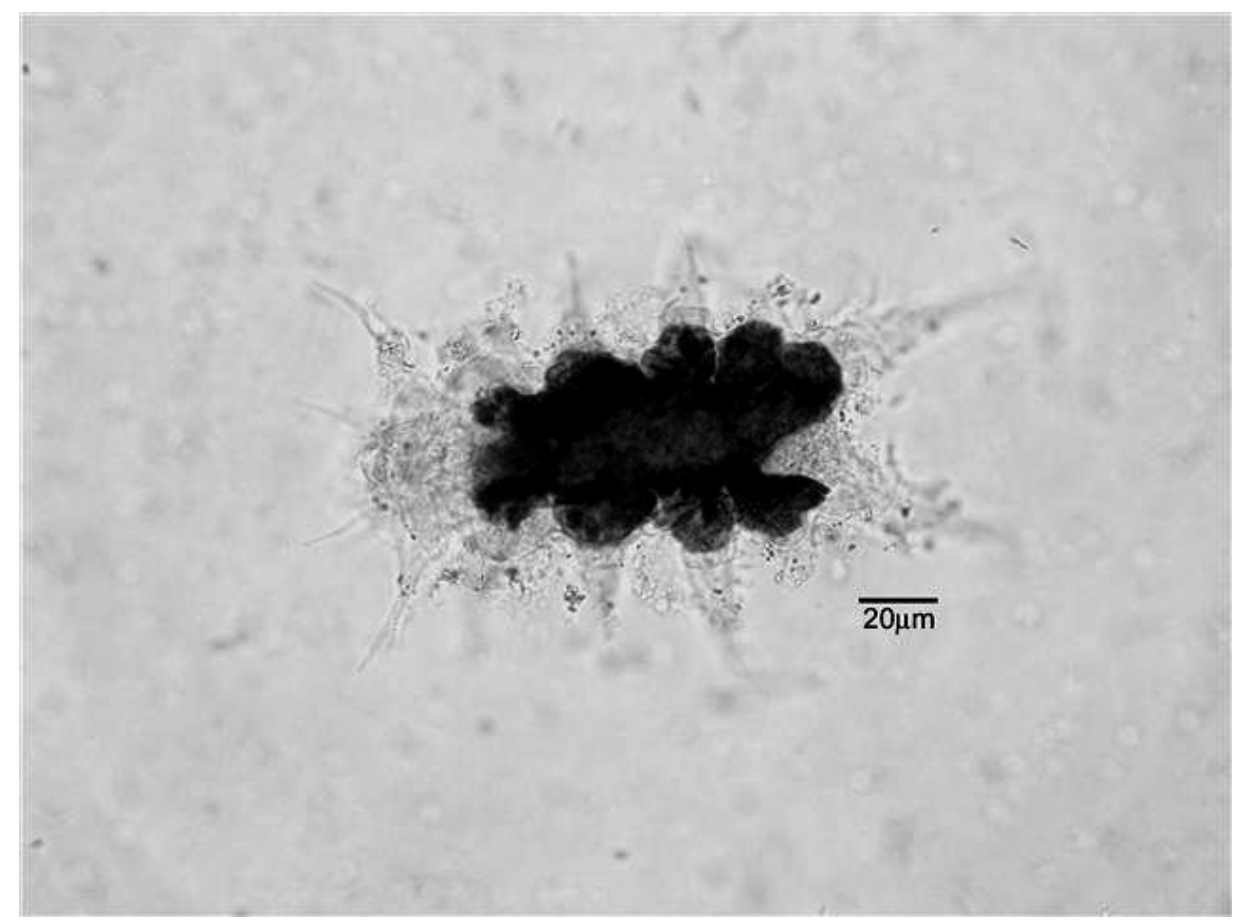

Figura 2 - Exemplar de Halechiniscus perfectus coletado no Arquipélago de São Pedro e São Paulo, RN, Brasil.

O Arquipélago de São Pedro e São Paulo consiste em ilhas rochosas situadas no hemisfério Norte sobre a Dorsal Meso-Atlântica, a cerca de $1.100 \mathrm{Km}$ da cidade de Natal - RN e 330 milhas náuticas do Arquipélago de Fernando de Noronha PE (Edwards \& Lubbock 1983, Amaral et al. 2002, 2006, 2009). Trata-se de um remoto grupo de ilhas próximo à linha do Equador, que ocupa uma área de aproximadamente $250 \mathrm{~m}$ por $426 \mathrm{~m}$, caracterizandose pela ocorrência de rochas plutônicas ultramáficas, que foram milonitizadas durante 0 seu posicionamento por forças tectônicas (Maia et al. 2001). O fundo da enseada é constituído por sedimentos provenientes da atividade biológica e da desagregação das rochas que constituem 0 arquipélago (Campos et al. 2005).

Foram coletadas 24 amostras de sedimentos nas profundidades de $6 \mathrm{~m}, 8 \mathrm{~m}, 12 \mathrm{~m}$ e $27 \mathrm{~m}$, utilizando-se um testemunhador com área de $10 \mathrm{~cm}^{2}$ (Higgins \& Thiel 1988). A extração e contagem da fauna foram feitas através de técnicas de rotina para meiofauna (Elmgren 1976). Os tardígrados foram transferidos para recipientes contendo uma solução de $75 \%$ de glicerol e $25 \%$ de formol a $10 \%$ por 10 minutos, sendo posteriormente montados em lamínulas contendo gotícula de glicerol puro em círculo de parafina e levados finalmente à vedação em forno quente, de acordo com técnicas descritas por Cobb (1917) e Renaud-Debyser \& Salvat (1963).

Stygarctidae Schulz, 1951 são Heterotardigrada que têm tipicamente cinco pares de apêndices cefálicos e um cirro mediano. Pernas sem dígitos, apresentando quatro garras com longos filamentos. São geralmente encontrados na superfície de areias grosseiras (Ramazotti \& Maucci 1983). O gênero Parastygarctus Renaud-Debyser, 1965 compreende cinco espécies que possuem uma placa cuticular cefálica, três placas dorsais e uma placa caudal. A cabeça é dividida em uma parte anterior com o cone bucal e o cirro interno, e uma parte posterior com os outros apêndices cefálicos. A clava anterior é alongada e as patas possuem quatro garras que portam longos filamentos. Dez exemplares de Parastygarctus sterreri (fig. 1) foram coletados em areias grossas biodetríticas às profundidades de $8 \mathrm{~m}, 12 \mathrm{~m}$ e $27 \mathrm{~m}$. Os exemplares examinados enquadram-se na descrição original da espécie, que alcança cerca de $170 \mu \mathrm{m}$ e possui cutícula com um ligeiro pontilhamento dorsal, caracterizando-se pela placa cuticular cefálica formando quatro grandes expansões, duas frontais e duas laterais, com o cirro mediano medindo $24 \mu \mathrm{m}$, papilas cefálicas entre 19 a $20 \mu \mathrm{m}$ e clava medindo 24 $\mu \mathrm{m}$ (Renaud-Mornant 1970a).

Esta família tem ocorrência referida para a Itália 
(Grimaldi de Zio et al. 1983a, 1983b, D’Addabbo Gallo et al. 1987, 1989, Gallo D'Addabbo et al. 1999, De Zio Grimaldi \& Gallo D’ Addabbo 2001), lugoslávia (De Zio 1965, Grimaldi de Zio et al. 1979, Renaud-Mornant \& Gourbault 1984), Malta (De Zio 1965), Madagascar (Renaud-Mornant 1975), Guadalupe (Renaud-Mornant \& Gourbault 1984), Martinica (Renaud-Mornant 1980), Bermudas (Renaud-Debyser 1965, Kristensen 1978, Kristensen \& Sterrer 1985), Galápagos (Matheus 1938) e EUA (Kristensen \& Higgins 1984), ocorrendo desde a região entre-marés até $80 \mathrm{~m}$ de profundidade.

Halechiniscidae Thulin, 1928 são Heterotardigrada que não apresentam placas cuticulares. Seus apêndices cefálicos são completos. As pernas apresentam cinco dígitos terminando em uma única garra cada. Habitam a superfície de sedimentos grosseiros, assim como Stygarctidae (Ramazzotti \& Maucci 1983). Foram encontrados 74 exemplares de Halechiniscus perfectus (fig. 2) associados a sedimento inconsolidado em todas as profundidades estudadas $(6 \mathrm{~m}, 8 \mathrm{~m}, 12 \mathrm{~m}$ e $27 \mathrm{~m})$. Todos os indivíduos examinados mostraram-se em conformidade com a descrição original da espécie, medindo cerca de $167 \mu \mathrm{m}$ (Schulz 1955). Apresentam apêndices cefálicos com uma parte basal grossa, de aspecto tubular. A abertura bucal é rostro-ventral, apresentando um estilete delgado. Os únicos apêndices cuticulares do corpo são dois espinhos situados dorsolateralmente na parte posterior. As patas aumentam progressivamente quanto ao comprimento, do $1^{\circ}$ ao $4^{\circ}$ par. A espécie tem ocorrência referida para a Itália (Schulz 1955, D’Addabbo Gallo et al. 1978, Grimaldi de Zio et al. 1980a, 1980b, 1982, 1983a, De Zio Grimaldi \& Gallo D’Addabbo 2001), Mediterrâneo (De Zio Grimaldi \& Villora-Moreno 1995, Villora-Moreno \& de Zio Grimaldi 1996, De Zio Grimaldi \& Gallo D'Addabbo 2001), França (Fize 1963, Renaud-Mornant 1970b, 1970c, Grimaldi de Zio et al. 1980b), Escócia (Morgan \& O'Reilly 1988), Ilhas Färoe (Hansen et al. 2001), Oceano Índico (Pollock 1976), Nova Caledônia (Renaud-Mornant 1967) e Polinésia (Salvat \& Renaud-Mornant 1969, Renaud-Mornant 1976) em médio e infralitoral até $170 \mathrm{~m}$.

Até 2006, somente 6 espécies de tardígrados marinhos tinham registro de ocorrência no Brasil: Batillipes pennaki Marcus, 1946, B. mirus Richters,
1909, B. tubernatis Pollock, 1971, Opydorscus fonsecae Renaud-Mornant, 1990, Echiniscoides sigismundi Schultze, 1865 e Orzeliscus belopus Marcus, 1952 (Verçosa et al. 2007). Recentemente, Da Rocha et al. (2009) registraram mais 6 gêneros e 6 espécies, encontrados no litoral de Pernambuco: Florarctus Delamare-Debouteville \& Renaud-Mornant, 1965, Raiarctus Renaud-Mornant, 1981, B. dicrocercus Pollock, 1970, B. annulatus De Zio, 1963, Mesostygarctus intermedius Renaud-Mornant, 1979, Dipodarctus subterraneus Renaud-Mornant, 1959, Stygarctus bradypus Schulz, 1951 e Wingstrandarctus intermedius Renaud-Mornant, 1967. Este é o primeiro registro de ocorrência de Parastygarctus sterreri Renaud-Mornant, 1970 e Halechiniscus perfectus Schulz, 1955 no Brasil, ampliando para 11 o número de gêneros e 14 o número de espécies de tardígrados marinhos no país.

\section{AGRADECIMENTOS}

Os autores agradecem a Alessandra Prates Botelho pela documentação fotográfica, ao CNPq pela concessão de bolsa de Iniciação Científica à primeira autora e financiamento do Projeto, bem como à Marinha do Brasil e à SECIRM pelo apoio logístico.

\section{LITERATURA CITADA}

AMARAL, FD, MM HUDSON, FL SILVEIRA, AE MIGOTTO, SM PINTO \& L LONGO. 2002. Cnidarians of Saint Peter and St. Paul Archipelago, Northeast Brazil. In: International Coral Reef Symposium, 9, Bali. Proceedings. v. 1, p. 567-572.

AMARAL, FD, SRM SILVEIRA, SL VASCONCELOS, \& CAC RAMOS. 2006. Biodiversidade de cnidários bentônicos. In: VASKE JÚNIOR, T.; LESSA, R.P.; NÓBREGA, M.F.; AMARAL, F.M.D.; SILVEIRA, S.R.M. Arquipélago de São Pedro e São Paulo: histórico e recursos naturais. Olinda: Livro Rápido. p. $42-55$.

AMARAL, FD, CMC DA ROCHA, CMR FARRAPEIRA, MS ALVES, SL PINTO, SMA LIRA, KK MELO, CAC RAMOS, ECL DOS SANTOS, JR MOURA, DAS OLIVEIRA, MM VERÇOSA, AVM MELO, APA OLIVEIRA, \& EF GONÇALVES. 2009. Distribuição espacial de invertebrados bentônicos infralitorais. In: O Arquipélago de São Pedro e São Paulo: 10 anos de Estação Científica. Brasília: SECIRM, v. 1, p. $126-134$.

CAMPOS, TFC, J DAS VIRGENS NETO, NK SRIVASTAVA, RA PETTA, LA HARTMANN, JFS MORAES, L MENDES \& SRM SILVEIRA. 2005. Arquipélago de São Pedro e São Paulo Soerguimento tectônico de rochas infracrustais no Oceano Atlântico. Ed. Sítios Geológicos e Paleontológicos do Brasil. Disponível em: http://www.unb.br/ig/sigep/sitio002/sitio002.pdf. Acesso em: 24/12/2005.

COBB, NA. 1917. Note on nemas. Contr. Sci. Nematol. n.5, p.117128.

DA ROCHA CMC, MM VERÇOSA, ECL DOS SANTOS, DF BARBOSA, DAS OLIVEIRA \& JRB SOUZA. 2009. Marine tardigrades from the coast of Pernambuco, Brazil. Meiofauna Marina, Vol. 17, pp. 97-101. 
DE ZIO, S. 1965. Distribuzione del mesopsammon in rapporto alla distanza dalla linea di riva ed alla distanza da un corso d'acqua salmastra. Boll. Zool. 32 (2): 525-537.

DE ZIO GRIMALDI, S \& M GALLO D'ADDABBO. 2001. Further data on the Mediterranean Sea Tardigrade Fauna. Zool. Anz. 240: $345-360$.

DE ZIO GRIMALDI, S \& S VILLORA-MORENO. 1995. Halechiniscus chafarinensis n. sp. (Halechiniscidae) a new marine tardigrada from the Alboran Sea (SW Mediterranean Sea). Cah. Biol. Mar. 36: 285-289.

D' ADDABBO GALLO, M, MR MORONE DE LUCIA, S GRIMALDI DE ZIO \& P GRIMALDI. 1978. Nuovi dati sui Tardigradi del Mesopsammon delle coste pugliesi. Thalassia Selentina 8: 63-66.

D' ADDABBO GALLO, M, MR MORONE DE LUCIA \& S GRIMALDI DE ZIO. 1987. Heterotardigrada of the Amendolara Shoal, High Ionian Sea. In: Bertolani, R. (ed.), Biology of Tardigrades. Selected Symposia and Monographs U.Z.I., I, Mucchi Modena pp. 103-110.

D' ADABBO GALLO, M, MR MORONE DE LUCIA \& S GRIMALDI DE ZIO. 1989. Two new species of the genus Styraconyx (Tardigrada: Heterotardigrada). Cah. Biol. Mar. 30: 17-33.

EDWARDS A \& R LUBBOCK. 1983. The ecology of Saint Paul's Rocks (Equatorial Atlantic). Journal Zool London, 200: 51-69.

ELMGREN, R. 1976. Methods of sampling sublittoral soft bottom meiofauna. Oikos, Suppl. v 15, p. 112-120.

FIZE, A. 1963. Contribution a L' étude de la microfaune des sables littoraux du Golfe d'aigües mortes. Vie Millieu 14 (4): 671-774.

GALLO D'ADDABBO, M, S DE ZIO GRIMALDI, MR DE LUCIA MORONE, R PIETANZA, R D'ADDABBO \& MA TODARO. 1999. Diversity and dinamics of an interstitial Tardigrada population in the Meloria Shoals, Ligurian Sea, with a redescription of Batillipes similis (Heterotardigrada, Batillipedidae). Ital. J. Zool. 66: 51-61.

GRIMALDI DE ZIO, S, MR MORONE DE LUCIA \& M D' ADDABBO GALLO. 1979. Osservazione su alcuni Tardigradi di una spiaggia pugliese e descrizione di Batillipes adriaticus sp. nov. (Heterotardigrada). Thalassia Selentina 9: 39-59.

GRIMALDI DE ZIO, S, M D' ADDABBO GALLO, MR MORONE DE LUCIA \& P GRIMALDI. 1980a. Ulteriori dati sui Tardigradi del mesopsammon di alcune spiagge pugliesi. Thalassia Selentina 10: 45-65.

GRIMALDI DE ZIO, S, MR MORONE DE LUCIA, M D' ADDABBO GALLO \& P GRIMALDI. 1980b. Conoscenze attuali sulla distribuzione dei Tardigradi marini nel Bacino del Mediterraneo. Mem. Biol. Mar. Oceanog., 10 Suppl. 6: 403-405.

GRIMALDI DE ZIO, S, M D' ADDABBO GALLO \& MR MORONE DE LUCIA. 1982. Neostygarctus acanthophorus n. gen. n. sp.: Nuevo tardigrado marino del Mediterraneo. Cah. Biol. Mar. 23: 319-323.

GRIMALDI DE ZIO, S, M D' ADDABBO GALLO \& MR MORONE DE LUCIA. 1983a. Marine Tardigrades of the "Secca dell' Armeleia" (Ioniann Sea), and redescription of Raiarctus colurus Renaud-Mornant 1981 (Heterotardigrada). Oebalia 9 N.S: 3342.

GRIMALDI DE ZIO, S, MR MORONE DE LUCIA, M D' ADDABBO GALLO \& P GRIMALDI. 1983b. Marine Tardigrades ecology. Oebalia 9 N. S.: 15-31.

HANSEN, JG, A JORGENSEN \& RM KRISTENSEN. 2001. Preliminary studies of the Tardigrade fauna of the Färoe Bank. Zool. Anz. 240: 385-393.

HIGGINS RP \& H THIEL. 1988. Introduction to the study of meiofauna. $1^{\text {a }}$ ed. Washington D.C.: Smithsonian Institution Press. 488 p.

KRISTENSEN, RM. 1978. Notes on Marine Heterotardigrades. 1. Description of two new Batillipes species, using the Eletron Microscop. Zool. Anz. 200 (1/2): 1-17.

Recebido: 06/08/2008

Aceito: 11/06/2009
KRISTENSEN, RM \& RP HIGGINS. 1984. A new family, Renaudarctidae, of Arthrotardigrada (Tardigrada: Heterotardigrada) from the East Coast of Florida, U.S.A. Trans. Amer. Micros. Soc. 103: 295-311.

KRISTENSEN, RM \& W STERRER. 1985. Phylum Tardigrada (water bears). In: Sterrer, W. (ed.), Marine flora and fauna of Bermuda. pp. 265-268.

MAIA, M, SICHEL SE, S ESPERANÇA \& J THIROT. 2001. The Equatorial Atlantic "cold spot": Constraints from osmium isotope composition, plate kinematics and tomography. EUG XI Marine Geology and Geophysics, 516.

MATHEWS, GB. 1938. Tardigrada from North America. Amer. Midl. Nat. 19: 619-627.

MORGAN, CI \& M O'REILLY. 1988. Additions to the Scottish tardigrade fauna, including a description of Megastigarctides setoloso new species, with a vised key for the identification of Scottish marine species. Glasg. Nat. 21 (4): 445-454.

POLLOCK, LW. 1976. Marine Flora and Fauna of the Northenastern United States. Tardigrada. NOAA Technical Report NMFS CIRC-394 pp. 1-25.

RAMAZZOTTI, G \& W MAUCCI. 1983. II phylum Tardigrada (III edizione riveduta e aggiornata). Mem. Ist. Ital. Idrobiol. 41:11012.

RENAUD-DEBYSER, J. 1965. Étude sur un Stygarctidé (Tardigrada) nouveau de Madagascar. Bull. Soc. Zool. France $90: 31-38$

RENAUD-DEBYSER, J \& B SALVAT. 1963. Récherches ecologiques sur la faune interstitielle des sables. Bassin d'Arcachon. Île de Bikini, Bahamas. Vie millieu. v. 14, p. 465550.

RENAUD-MORNANT, J. 1967. Tardigrades de la Baie Saint-Vicent Nouvelle Calédonie. In: Exp. Fr. Récifs Coral Nouvelle_Calédonie. Ed. Fondation Singer-Polignac, Paris 12: 103-118.

RENAUD-MORNANT, J. 1970a. Parastygarctus sterreri n. sp. Tardigrade marin nouveau de I' Adriatique. Cah. Biol. Mar. XI: 355-360.

RENAUD-MORNANT, J. 1970b. Campagne d' essays du "Jean Charcot” (3-8 Décembre 1968) 8; Méiobenthos. II. Tardigrades. Cah. Biol. Mar. 2ª Série 42 (5): 957-969.

RENAUD-MORNANT, J. 1970c. Tardigrades marins des Bermudes. Cah. Biol. Mar. 2a Série 42: 1268-1276.

RENAUD-MORNANT, J. 1975. Occurrence of the genus Tanarctus Renauld-Debyser 1959 in North Eastern Atlantic Waters with a description of $T$. ramazzotti. n. sp. (Arthrotardigrada). Mem. Ist. Ital. Idrobiol., 32 Suppl.: 325-332.

RENAUD-MORNANT, J. 1976. Tardigrade marins de Polynesie. Cah. Pacific, 19: 289-297.

RENAUD-MORNANT, J. 1980. Description de trois espèces nouvelles du genre Tanarctus Renaud-Debyser, 1959, et creation de la sous-famille des Tanactineae, subfam. nov. (Tardigrada, Heterotardigrada). Bull. Mus. Natn. Hist. Nat. Paris, 4⿳亠丷a sér., 2, A (1): 129-141.

RENAUD-MORNANT, J \& N GOURBAULT. 1984. Premiéres prospections meiofaunistiques en Guadaloupe. Hydrobiologia 118: 113-118.

SALVAT, B \& J RENAUD-MORNANT. 1969. Étude écologique du macrobenthos et du meiobenthos d' un fond sableux du lagon de Mururoa (Tuamotu-Polynesia). Cah. Pacif. 13: 159-179.

SCHULZ, E. 1955. Studien an marinen Tardigraden. Kieler Meeresforsch: XI, (1), 74-79.

VERCOSA, MM, DAS OLIVEIRA, DF BARBOSA, ECL SANTOS, JRB SOUZA \& CMC DA ROCHA. 2007. Tardígrados marinhos do litoral de Pernambuco - Brasil. In: XII Congresso LatinoAmericano de Ciências do Mar. XII COLACMAR - Resumos expandidos.

VILLORA-MORENO, S \& S DE ZIO GRIMALDI. 1996. New records of marine Tardigrada in the Mediterranean Sea. Zool. J. Linnean Society 116: 149-166. 